\title{
A97-32484
}

AIAA-97-2024

\section{A local Navier-Stokes preconditioner for all Mach and cell Reynolds numbers}

\author{
Dohyung Lee* ${ }^{*}$ and Bram van Leer ${ }^{\dagger}$ \\ Department of Aerospace Engineering, \\ The University of Michigan, Ann Arbor, MI 48109-2118 \\ John F. Lynn ${ }^{\ddagger}$ \\ Ford Motor Company, Dearborn, MI 48121
}

\begin{abstract}
A new family of optimal Euler preconditioners is presented that may resolve the stability problem associated with stagnation points. These preconditioners produce a close-to-orthogonal eigenvector system and are less sensitive to flow-angle variation across cells. Single- and multi-grid benefits of Euler preconditioning are presented and illustrated by some numerical results. Navier-Stokes preconditioning can be extended from the Euler technique with two methods: (a) by using block-Jacobi preconditioning for the viscous terms; (b) by introducing analytic dependence on the cellReynolds number in the preconditioner. Block-Jacobi preconditioning can also be applied to turbulent viscous flow calculations for a proper rescaling of the source terms. With these techniques it is possible to produce a local Navier-Stokes preconditioner effective for all Mach and cell-Reynolds numbers. Both two methods are illustrated with numerical tests.
\end{abstract}

\section{Why use local precondition- ing?}

For a Navier-Stokes code to converge fast it is necessary (but not sufficient) that the embedded Euler code converges fast, too. In working toward more efficient Navier-Stokes codes it therefore is useful to stay informed on Euler methods, and exploit the latest advances in that area.

\footnotetext{
*Postdoctoral Fellow, Member AIAA

tProfessor, Fellow AIAA

¿Researcher

Copyright (C) 1997 by the American Institute of Aeronautics and Astronautics, Inc. All rights reserved.
}

During the 1990s our understanding of the Euler convergence process has significantly increased. There is mounting evidence that the treatment of the true advection equations hidden in the Euler system (e.g. advection of entropy) has to be different from that of the coupled acoustic equations arising in subsonic flow. Whether starting from the equations in conservation form or primitive variables, this calls for a suitable decomposition of the system, in which the embedded acoustic equations are isolated from the advection equations.

Ta'asan [22] suggests using a set of "canonical" equations based on the steady form of the Euler equations; this has its roots in vintage rules prescribed by Brandt in his 1984 Multi-Grid Guide [3]. In Brandt's work the emphasis is on achieving the theoretical limit of multigrid convergence, and not necessarily on the accuracy of the spatial operator.

In contrast, Roe and Mesaros [18, 17] searched for a genuinely multi-dimensional splitting of the spatial Euler operator ("fluctuation splitting") that would match accuracy to compactness. They ended up discretizing of the system that results from applying the local preconditioning of Van Leer, Lee and Roe [28]. The most advanced applications of these preconditioned equations are by Deconinck et al. [7], and use the so-called matrix form of the PSI advection scheme, which automatically distinguishes between omnidirectional acoustic wave propagation and unidirectional advection.

The combination of the preconditioned equations and the fluctuation-split scheme, formulated on unstructured triangular or tetrahedral grids, differs in essence from standard Euler schemes. Such a nu- 
merical strategy will not easily be adopted by the larger CFD community, which has a considerable effort invested in higher-order Godunov-type methods with TVD limiters. Fortunately, it has been demonstrated that the use of the preconditioned equations ${ }^{1}$ alone, without any essential modification of the discretization ${ }^{2}$, has a large potential pay-off. In addition to providing the decoupling for implementing fluctuation splitting, proper local preconditioning can have the following major, well documented, benefits.

1. It removes from the Euler equations the stiffness caused by the range of characteristic speeds, thus improving the convergence rate of any marching scheme $[28,12,10,27]$;

2. It makes the system behave more like a scalar equation, thus facilitating the design of effective auxiliary techniques, such as multi-grid smoothers $[14,15,13,16]$, residual smoothing [13], approximate factorization [11];

3 . It produces discretizations that remain accurate in the limit of vanishing Mach number $[28,12,10$, $26,27,21,11]$.

In short, there are single-grid-relaxation, multi-gridrelaxation and accuracy benefits to the simple explicit technique of local preconditioning. Those who are not taking advantage of these are selling themselves short. In Section 2 we show some numerical results in support of the above benefits.

The extension of local preconditioners from the Euler to the Navier-Stokes equations is not trivial. The wave speeds now become complex as a result of the dissipative terms, which greatly complicates any analysis, even when carried out with symbolic manipulation. A new parameter, the Reynolds number, appears in the equations; in their discretized form the key quantity is the cell Reynolds number $R e_{h}$. For medium $(\approx 1)$ to high values of $R e_{h}$ there is little need to deviate from the Euler preconditioner $[11,27]$, but for lower values of $R e_{h}$ this "Euler approach" fails.

In Section 4, we present the design analysis for a Navier-Stokes preconditioner that works for all values

\footnotetext{
${ }^{1}$ If the 2-D Euler equations are written as $\mathbf{U}_{t}+\mathbf{A} \mathbf{U}_{x}+\mathbf{B} \mathbf{U}_{y}=$ $\mathbf{0}$, the preconditioned equations read $\mathbf{U}_{t}+\mathbf{P}\left(\mathbf{A} \mathbf{U}_{x}+\mathbf{B} \mathbf{U}_{y}\right)=\mathbf{0}$ or $\mathbf{P}^{-1} \mathbf{U}_{t}+\mathbf{A} \mathbf{U}_{x}+\mathbf{B} \mathbf{U}_{y}=\mathbf{0}$.

${ }^{2}$ Besides multiplication of the residual by the preconditioning matrix, the artificial-dissipation matrices must be modified, so that they are appropriate for the preconditioned equations. If the flux Jacobian is $A$, the corresponding dissipation matrix is some average of $|\mathbf{A}|$, and the preconditioning matrix $P$, then the dissipation matrix must be changed into $\mathbf{P}^{-1}|\mathbf{P A}|$. This means that after preconditioning the dissipation matrix becomes $|\mathbf{P A}|$, which matches the preconditioned coefficient matrix PA.
}

of $M$ and $R e_{h}$. Numerical tests illustrating the performance of this preconditioner are presented in Section 5.

\section{Benefits of Euler precondi- tioning}

\subsection{Effect on single- and multi-grid re- laxation}

As mentioned above, the equalization of wave speeds, achieved by optimal Euler preconditioners, removes the stiffness encountered in steady-state calculations (especially explicit calculations), yielding convergence acceleration already on a single grid. Furthermore, the concentration of discrete eigenvalues, achieved by optimal Euler preconditioners, makes it possible to design multistage marching schemes with optimal highfrequency damping regardless of flow angle and Mach number; these are particularly suited for multigrid relaxation on semi-coarsened grids. The single-grid and multigrid convergence-acceleration mechanisms are independent, so their individual effects add up; this was demonstrated conclusively by Tai [23] for 1-D Euler schemes and Lynn [13] for 2-D Euler schemes. Some of Lynn's results were presented at the previous AIAA CFD Conference; below we present a different sample, intended to drive the message of the double benefit home once more.

Another message worth repeating [16] is that the local preconditioning matrix resulting from retaining only the main-diagonal block of an implicit upwind Euler discretization (point/Jacobi relaxation) does not have any single-grid acceleration effect. Point/Jacobitype preconditioning is currently enjoying a renewed interest $[2,20]$ because of its smoothing effect on highfrequency error combinations, useful in multigrid relaxation with semi-coarsening; for this reason it was already recommended by Mulder [19].

Table 1 illustrates the compound single/multigrid benefit for a set of second-order-accurate calculations of inviscid subsonic flow $\left(M_{\text {inflow }}=0.35\right)$ in a $2 D$ channel with a circular bump on one wall (1979 GAMM Workshop parameters: thickness $4.2 \%$ of chord, height 2 chords). A high-resolution spatial discretization ( $\kappa=$ 0 , Van Albada limiter, Roe's Riemann solver) was used; time marching was done with a 4-stage scheme optimized for best damping of all high $x$ - and $y$-frequency combinations, as needed for semi-coarsened multigrid relaxation. A "design graph" for this scheme, i. e. the optimally scaled Fourier footprint of the spatial dis- 
cretization plotted on top of the scheme's stability domain and amplification-level lines, is shown in Figure 1.

The two things to be learned from Table 2 (work needed for residual reduction) are:

(a) Local matrix preconditioning yields faster convergence than local time-stepping ( $\equiv$ scalar preconditioning) on a single grid. In this subsonic case the speed up is not so impressive, as the singlegrid convergence process is dominated by acoustic waves bouncing between the walls; these are not affected by the preconditioning.

(b) The maximum gain from multigrid relaxation is greater for local matrix preconditioning than for local time-stepping.

A contrasting aspect is provided by Table 2 , which contains the convergence data for a supersonic flow case $\left(M_{\text {inflow }}=1.4\right)$. This set clearly brings out the singlegrid benefit: the single-grid acceleration by matrix preconditioning is so strong that adding more grid levels yields little additional speed-up.

In the next test problem the initial values consist of a uniform state (flow angle $=10^{\circ}$, range of $M$ values) with random perturbations on a $32 \times 32$ uniform grid; at the boundaries free-stream conditions are prescribed. A first-order upwind spatial operator with optimized four-stage time-stepping was used. Table 3 shows work needed till convergence for various relaxation strategies: single-grid with local time-stepping (SG-LTS), single-grid with matrix time-stepping (SGMTS), regular multigrid with local time-stepping (MGLTS), regular multigrid with matrix time-stepping (MG-MTS). Both local time-stepping methods are affected by the variation of condition number with Mach number, whereas the matrix time-stepping methods are virtually insensitive to Mach number.

The above acceleration can still be enhanced at little computational cost by introducing explicit residual smoothing, which significantly increases the allowable CFL number without compromising highfrequency damping. Owing to the preconditioning the smoothing is equally effective for all physical modes (acoustic and advective). Figure 2 shows a design graph for the preconditioned 4-stage first-order upwind scheme when explicit residual smoothing, with coefficient $\epsilon=0.1$ is added. The effect of the residual smoothing is an increase of the optimized CFL number from 2.64 to 3.63 , without sacrificing high-frequency damping. The improved convergence is clear upon comparing Table 4 (perturbation test, work needed using explicit residual smoothing) with Table 3.

\subsection{Effect on accuracy at low Mach number}

One unexpected side effect of certain preconditioners, such as those of the Van Leer-Turkel family, is that they prevent the deterioration of accuracy encountered when computing flows at ever-decreasing Mach number on a fixed grid, a phenomenon first reported by Volpe [31]. This beneficial and very useful effect of local preconditioning is owing to the modification of the artificial-dissipation matrices that has to be implemented anyway for stability reasons. This required modification was originally considered a nuisance, since it makes preconditioning more intrusive; now we know that codes for compressible flow actually need such a modification if they are to be applied to problems in which incompressible and compressible flow occur side by side. Such situations arise, for instance, in propulsion [4], in low-speed high-lift flow [27] and in V/STOL maneuvering [21]. Local preconditioning makes it possible to solve flow problems of this kind with a single code, overcoming both the loss of accuracy and the loss of convergence speed, not a small accomplishment for a modest explicit technique.

Since its discovery $[28,12]$, the accuracy benefit has been demonstrated over and over in numerical tests $[28,12,10,26,27,18,17,21,7,11]$; we need not repeat any of these here. An important development, still worth emphasizing, is that the effect has also been explained with mathematical analysis. The asymptotic analysis by Turkel [26] et al. provides the detailed structure a preconditioning matrix must have in order to properly balance the artificial dissipation terms with the inviscid flux terms for $M \rightarrow 0$ (lack of balance causes the loss of accuracy). Reed's [21] analysis is based on the modified equation and is less detailed; it shows that the truncation error of the discrete pressure equation is multiplied by $M^{2}$ owing to the preconditioning.

An important observation based on the analysis of Turkel et al. is that point/Jacobi-type preconditioning does not have the structure needed for accuracy preservation in the incompressible limit. This is clear from its common use, which does not include any change to the artificial-dissipation matrices. However, even if it would be used to modify the latter, this would not properly balance the terms in the scheme when $M$ approaches 0.

The analysis in [26] does not answer the question if a local loss of accuracy can result in a very limited region of low Mach number, such as a stagnation region, if no preconditioning is used. We suspect there indeed is a local loss of accuracy, but there has been 


\begin{tabular}{|c|c|c|c|c|c|c|c|c|c|}
\hline & & & & & ber of & tages & & & \\
\hline & & Lor & al tim & -step & ing & Mat & $x$ pre & ondit & oning \\
\hline & & 3 & 4 & 5 & 6 & 3 & 4 & 5 & 6 \\
\hline No. & 1 & 3702 & 3740 & 3685 & 3744 & 3126 & 2976 & 2380 & 2916 \\
\hline of & 2 & 2241 & 1622 & 1284 & 1087 & 1366 & 1271 & 780 & 838 \\
\hline grid & 3 & 692 & 582 & 582 & 583 & 388 & 301 & 632 & 615 \\
\hline levels & 4 & 755 & 751 & 705 & 708 & 319 & 191 & 194 & 219 \\
\hline
\end{tabular}

Table 1: Work units required to reduce $\|T E\|_{1}$ to $5 \times 10^{-2}$; channel flow, $M=0.35 ; 64 \times 32$ grid. Defectcorrection cycles; nested iteration for initial guess.

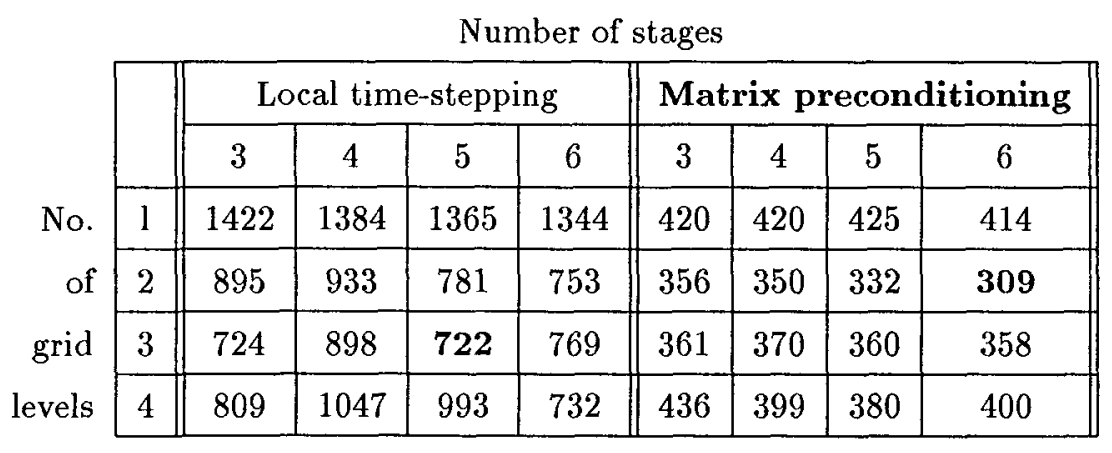

Table 2: Same as Table 2, but for $M=1.4$.

no study to confirm this. If proven correct, it might have far-reaching consequences for our trust in standard discretizations.

\section{Advances in Euler precondi- tioning}

One serious problem associated with the use of local preconditioning, even if it does the right thing in the limit of incompressibility, is that it commonly breaks down locally when the Mach number vanishes, i. e. in a stagnation point. This break-down manifests itself in non-convergence, if not instability. At the previous AIAA CFD Conference two papers were presented adressing different causes of this problem:

1. Sensitivity of the preconditioning matrix to the flow angle for $M \rightarrow 0$ [29], an point of concern particularly for the Van Leer-Lee-Roe matrix;
2. Degeneration of the eigenvector system of the preconditioned equations for $M \rightarrow 0$ [?], a problem plaguing most preconditioners currently in use.

Of these factors, the latter is by far the most serious.

To reduce the flow-angle sensitivity, the authors of the first paper chose to allow an increase of the condition number from 1 to 2 ; this at least made a nonconservative flow code converge to an accurate low-speed solution, which previously had been unattainable [18]. As a "fix" for eigenvectors becoming parallel ${ }^{3}$, Darmofal and Schmid suggested to limit the value of $M$ in the preconditioner from below, for instance,

$$
M_{\lim }=\max \left(M, \epsilon M_{\infty}\right),
$$

where $\epsilon$ is a small fraction; this was shown to cure even conservative schemes. The necessity of such limiting had already been found in practice by Turkel [25], although Turkel attributed it to the loss of symmetriz-

\footnotetext{
${ }^{3}$ The original system has a fully orthogonal eigenvector structure.
} 


\begin{tabular}{|c||c|c|c|c|c|c|c|c|c||}
\multicolumn{10}{|c}{ Mach Number } \\
\hline & 0.1 & 0.35 & 0.5 & 0.85 & 0.99 & 1.01 & 1.2 & 2.0 & 5.0 \\
\hline SG-LTS & 1224 & 408 & 352 & 984 & 1512 & 1552 & 784 & 272 & 164 \\
\hline SG-MTS & 280 & 272 & 272 & 272 & 224 & 188 & 160 & 148 & 128 \\
\hline MG-LTS & 996 & 401 & 263 & 263 & 816 & 1757 & 290 & 252 & 228 \\
\hline MG-MTS & 76 & 76 & 83 & 83 & 83 & 83 & 83 & 90 & 103 \\
\hline
\end{tabular}

Table 3: Work required to reduce residual norm by $10^{-5}$; perturbed uniform flow, $\phi=10^{\circ}$.

Mach Number

\begin{tabular}{|c||c|c|c|c|c|c|c|c|c||}
\hline & 0.1 & 0.35 & 0.5 & 0.85 & 0.99 & 1.01 & 1.2 & 2.0 & 5.0 \\
\hline SG-LTS & 688 & 204 & 196 & 556 & 848 & 916 & 436 & 152 & 108 \\
\hline SG-MTS & 176 & 172 & 168 & 168 & 136 & 116 & 96 & 88 & 72 \\
\hline MG-LTS & 760 & 256 & 208 & 166 & 650 & 1438 & 180 & 180 & 187 \\
\hline MG-MTS & 62 & 62 & 62 & 69 & 69 & 69 & 69 & 76 & 83 \\
\hline
\end{tabular}

Table 4: Same as Table 4, but with the use of explicit residual smoothing $(\epsilon=0.1)$.

ability of the system after preconditioning. Unfortunately, the appearance of two different Mach numbers considerably complicates the analysis and design of any but the simplest preconditioners, as well as the construction of the corresponding artificial-dissipation matrices; the powers of symbolic manipulation are easily exhausted. In addition, the choice of the cut-off value for $M$ is problem-dependent; a "safe" larger value of $M_{\text {lim }}$ may noticeably slow down convergence.

We therefore are pleased to report here - with guarded optimism - that we have found, and are investigating, a group of preconditioning matrices suffering much less from the above eigenvector degeneration. Specifically, eigenvectors related to plane waves moving in the same direction are non-parallel in the limit of $M \rightarrow 0$; many can actually be made orthogonal, and some even for the entire Mach-number range. Among these matrices there may be one that does not require the Darmofal-Schmid fix.

Some of these matrices are members of the same family that includes the Van Leer-Lee-Roe and Turkel matrices. To fix our thoughts, let us assume that the Euler equations are expressed in terms of the symmetrizing variables $\mathbf{U}$, defined by $d \mathbf{U}=$ $(d p / \rho a, d u, d v, d w, d S)$, and that the flow is aligned with the positive $x$-axis. Using the notation $\beta=$ $\sqrt{1-M^{2}}$ for $M<1$, the Van Leer/Turkel family with free parameter $f$ has the generic two-dimensional form

$$
P_{V L, 96}=\left(\begin{array}{cccc}
\frac{M^{2}}{\beta} & -\frac{M}{\beta} f & 0 & 0 \\
-\frac{M}{\beta} & 1+\frac{f}{\beta} & 0 & 0 \\
0 & 0 & \beta & 0 \\
0 & 0 & 0 & 1
\end{array}\right)
$$

For $f=1$ this is the Van Leer-Lee-Roe matrix, for $f=0$ a version of the Turkel matrix, made optimal for the transonic flow regime. If, for small $M$, we choose $f$ close to -1 , i. e.

$$
f \approx-1+r M^{2}
$$

it is seen that the element $P_{22}$ becomes small:

$$
P_{22} \approx\left(r-\frac{1}{2}\right) M^{2}
$$

This form can still be positive-definite, and has the improved eigenvector structure mentioned earlier.

We first experimented with the case $r=-\frac{1}{2}$; the matrix in question was developed by $D$. Lee [11] with 
the purpose of removing any flow-angle dependence ${ }^{4}$. This matrix, presumably suitable for computing stagnating flow, reads:

$P_{\text {stagnation }}=\left(\begin{array}{cccc}M^{2} & M \sqrt{1+M^{2}} & 0 & 0 \\ -M \sqrt{1+M^{2}} & \left(b_{0}-1\right) M^{2} & 0 & 0 \\ 0 & 0 & 1 & 0 \\ 0 & 0 & 0 & 1\end{array}\right)$

where $b_{0}=0$ gives the optimal wave pattern for all $M<1$, but $b_{0}>1$ is needed for positive definiteness of $\mathbf{P}$ and symmetrizability of the preconditioned equations. In the limit of low Mach number, this preconditioner constructs a perfectly orthogonal eigenvector structures for waves moving in the streamwise direction and non-parallel eigenvectors for waves moving in the direction normal to the flow.

The performance of this preconditioning can be judged from the converged velocity fields shown in Figure 3: the stagnation preconditioner can successfully compute stagnating flow in the half-plane. The corresponding convergence histories are shown in Figure 4. Note the absence of convergence without preconditioning, and the instability caused by Van Leer preconditioning.

However, the new preconditioner is not perfect either: when dropping the solid-wall reflection condition in an attempt to compute a full-plane stagnation-flow, the new preconditioner generated large amounts of vorticity, preventing convergence. For the larger Mach numbers in transonic regions, the stagnation preconditioner loses its effectiveness due to a severe violation of admissibility conditions $\left(b_{0}=0\right)$ or an improper wave pattern $\left(b_{0}>0\right)$.

A variant of the stagnation preconditioner can be smoothly linked to the Van Leer-Lee-Roe preconditioner, the best performer at $M=1$. The new stagnation-friendly all-purpose preconditioner follows the matrix structure of (2) with $f$ defined by

$$
f=\frac{\beta-2 M^{2}}{1+M^{2}} \text {. }
$$

This new preconditioner produces perfectly orthogonal eigenvectors for waves moving in the streamwise direction for all Mach numbers, as well as satisfaction of symmetrizability and positivity conditions. In spite of the complicated form of (6), the numerical implemenation of this matrix is quite simple because any matrix

${ }^{4}$ This matrix is valid for an arbitrary flow angle; it needs no rotational similarity transformation! of the form (2) produces simply structured artificialviscosity matrices, independent of $f$. We repeat that the "fix" (1) complicates the construction of artificialviscosity matrices. In the stagnation-flow test of Figure 3 , this preconditioner was also robust and accelerated convergence as expected.

\section{From Euler to Navier-Stokes Equations with turbulence modeling}

For the design of Navier-Stokes preconditioners it suffices to consider the linearized equations. In two dimensions these can be written as

$$
\mathbf{U}_{t}=L_{\mathrm{Eu}} \mathbf{U}+\mathbf{C} \mathbf{U}_{x x}+\mathbf{D} \mathbf{U}_{x y}+\mathbf{E} \mathbf{U}_{y y} .
$$

The first term on the right-hand side is the spatial Euler operator; the remaining terms are viscous/conductive. In the discrete version the latter are approximated by central differencing.

Two ways of extending local Euler preconditioners to the Navier-Stokes equations have been reported in the literature:

1. Adding to the Euler preconditioner the viscous/conductive entries arising in block-Jacobi preconditioning; this technique is due to Godfrey $[8,10,9]$;

2. Introducing cell-Reynolds-number dependence in the entries of the Euler preconditioner according to a Navier-Stokes dispersion analysis, a technique due to Venkateswaran et al. [30]

With regard to the first technique: the objections to Jacobi-type preconditioning for the Euler residual are not valid for the Navier-Stokes terms, because these terms by themselves are very well conditioned ${ }^{5}$. The main effect of Jacobi preconditioning when used only for the viscous/conductive terms is: rescaling the dissipative scales with respect to the convection scales [11]. This is achieved in practice by fixing the highestfrequency eigenvalues (represented in the Fourier footprint by the left-most negative-real point), regardless of Mach or cell-Reynolds number:

$\mathbf{P}_{\mathrm{NS}}^{-1}=\mathbf{P}_{\mathrm{Eu}}^{-1}+\frac{2}{\alpha}\left(\frac{\mathbf{C}}{(\Delta x)^{2}}+\frac{\mathbf{E}}{(\Delta y)^{2}}\right), \quad \alpha=\frac{q(\beta+\mathcal{A R})}{\Delta x}$

${ }^{5}$ The dissipative time-scales do not differ more than a factor $\max \left(\gamma / P_{r}, 4 / 3\right)$. 
here $q$ is the flow speed. This formula is valid for firstorder upwind differencing of the Euler terms, subsonic case; for a higher-order Euler discretizations like a $\kappa$ scheme, a correction factor $1-\kappa$ to the Euler contribution is required [11]. A strong point in favor of this composite way of preconditioning is that it properly incorporates the cell geometry, and can handle the very large cell-aspect ratios needed in turbulent boundarylayer calculation $\left(\mathcal{A R} \approx 10^{5}\right.$ ). The idea of rescaling through preconditioning can easily be extended to include source-term rescaling, and is in essence the same as the point-implicit treatment favored for stiff source terms. This makes Jacobi-type Navier-Stokes preconditioning suitable for PDE-based turbulence modeling, which require the capacity to deal with stiff source terms as well as large cell-aspect ratios. If the sourceterm vector on the right-hand side is $\mathbf{H}$, the preconditioner for the extended system of equations takes the form

$$
\mathbf{P}_{\mathrm{NS}, \mathrm{turb}}^{-1}=\mathbf{P}_{\mathrm{Eu}}^{-1}+\frac{1}{\alpha}\left(\frac{2 \mathbf{C}}{(\Delta x)^{2}}+\frac{2 \mathbf{E}}{(\Delta y)^{2}}-\Delta t \frac{\partial \mathbf{H}}{\partial \mathbf{U}}\right)
$$

Figures 5 and 6 show how the Fourier footprint for this type of system is affected by the preconditioner. In Figure 5 (unpreconditioned) notice that some eigenvalues, due to the presence of the source terms, have moved to a point on the negative real axis outside the main locus; this reduces the allowable time step. In Figure 6 (preconditioned) these values have been scaled back.

Unlike Euler preconditioners, composite NavierStokes preconditioners of the above type are simplest when appearing as $\mathbf{P}^{-1}$. Analytical inversion is not attractive, so the method becomes truly point-implicit.

The second technique requires a Fourier analysis of the linearized equation and subsequent eigenvalue computation; to do this analytically is much harder than for the Euler equations. In spite of symbolic computing, complete results have been obtained only for the unpreconditioned equations in one dimension, under simplifying assumptions. The effect of preconditioning can only be predicted in certain asymptotic cases ( $M$ and/or $R e$ large or small); evaluating the performance of a proposed preconditioner requires numerical calculation of eigenvalues.

The eigenvalues coming out of a Navier-Stokes dispersion analysis are complex, with the imaginary part representing propagation and the negative real part damping. The condition number still is defined as the ratio of the largest and smallest moduli of eigenvalues, and properly takes into account both physical effects. We therefore continue to pursue the optimization of the condition number. Using educated guessing and symbolic manipulation we have developed a family of 1-D Navier-Stokes preconditioners that is capable of connecting the Van Leer-Lee-Roe Euler preconditioner to each of the two distinct asymptotic viscous regimes described below. Using the symmetrizing state variables listed earlier ${ }^{6}$ we can write this matrix family as

$$
\mathbf{P}_{v l, \text { Explicit }}=\left(\begin{array}{ccc}
P_{11} & -M Q & \frac{M^{2} Q-P_{11}}{\rho a} \\
-M Q & Q+1 & 0 \\
0 & 0 & 1
\end{array}\right),
$$

where $P_{11}$ and $Q$ must have the following asymptotic values: [30]:

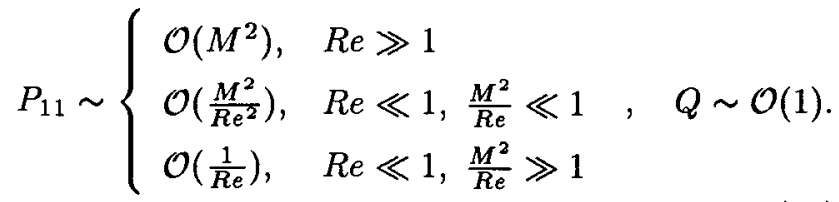

The three asymptotic regimes are, respectively, the inviscid or Euler limit, the acoustic-dominated viscous limit, and the viscosity-dominated limit.

The two viscous regimes are different in severity of viscous damping. In the acoustic-dominated limit both acoustic waves are undamped, so that there is only one damped mode; in the viscosity-dominated limit there are two damped modes ${ }^{7}$. (NB: For the unpreconditioned equations the distinction between the two viscous limits is governed by $M / R e$ rather than $M^{2} / R e$.)

It should be mentioned that the Reynolds number used in the dispersion analysis is always based on the wave length of the Fourier mode considered. When using the results of this analysis for the formulation of a $R e$-dependent preconditioner, $R e$ must be interpreted as the cell-Reynolds number $R e_{h}$.

Inserting $Q=0$ produces the equivalent of Chorin's ${ }^{8}$ preconditioner, used initially by Turkel [24] and preferably by Merkle et al. [4].

One problem with this preconditioner is that, according to the PDE analysis, it creates a small positive growth rate for one wave mode [30]. In practice this mode may be suppressed by the artificial dissipation present in the discretization, in particular if the

\footnotetext{
${ }^{6}$ The symmetrizing variables defined previously by $d \mathbf{U}=$ $(d p / \rho a, d u, d v, d w, d S)$ are sometimes called the "Euler symmetrizing variables," and actually are not the best choice for a Navier-Stokes analysis. The "Navier-Stokes symmetrizing variables," with $d \mathbf{U}=(a d p / \sqrt{\gamma} \rho, d u, d v, d w, a d T / \sqrt{\gamma(\gamma-1)} T)$ symmetrize all coefficient matrices in the Navier Stokes equations [1].

${ }^{7}$ There is always one undamped mode corresponding to the continuity equation, which carries no viscous terms.

${ }^{B}$ Named after Chorin, because it relates to his artificialcompressibility method [5].
} 
marching scheme is implicit. This, of course, is does not relieve us of the duty to search for better explicit preconditioners. It is conceivable that a smarter choice of the matrix elements will eventually remove the growing mode, and that the analysis can be extended to multidimensional preconditioning.

We have found that the growing mode can be removed by combining the result of the dispersion analysis with the addition of the viscous Jacobi block; this has produced a useful 2-D preconditioner. Specifically, we have modified the $(1,1)$ element in the Van LeerLee-Roe preconditioner (cf. Eq. (2)) according to the branched expression of Eq. (11), and then added the viscous Jacobi block, as in Eq. (8). The value of $\alpha$ must be changed correspondingly. Details can be found in [11] and will be presented in the final paper.

Table 5 shows the condition number achieved by a variety of Navier-Stokes preconditioners in the three asymptotic regimes distinguished in Eq. (11). Only their 1-D versions are considered, as a multidimensional analysis so far has not appeared possible.

For the original Navier-Stokes equations, the stiffness in the Euler limit is independent of $R e$ and increases as the Mach number decreases; for viscositydominated flow, on the other hand, it is independent of $M$, increasing when $R e$ decreases. For acousticdominated viscous flow the stiffness varies with both $M$ and $R e$. The use of preconditioning completely changes this pattern. The aim is to make the condition number equal to 1 or $O(1)$; this has been achieved using Eqs. $(10,11)$ (but remember the growing mode), and there is hope that the Jacobi-type preconditioners can be improved up to this mark.

The listings in Table 5 are illustrated in detail by the following four carpet plots, Figures 7-11, based on numerically obtained eigenvalues. Figure 7 shows the condition number for the unpreconditioned 1-D Navier-Stokes equations as a function of Mach number $\left(10^{-4} \leq M \leq 10^{-1}\right)$ and cell-Reynolds number $\left(10^{-5} \leq R e \leq 10^{5}\right)$. The number is seen to increase beyond bound for vanishing $M$ or $R e$. Figure 8 shows that the Euler preconditioner of Van Leer-Lee-Roe creates a large usable domain, $R e \geq 1$, larger than the Euler domain. The same is true for Turkel's preconditioner, see Figure 9. This explains results recently reported by Turkel et al. [27], viz. that the Euler preconditioner was effective in 2-D and 3-D viscous flow computations. The cell-Reynolds number in these calculations nowhere dropped below 1, not even in the most stretched boundary-layer cells.

Figures 10 and 11 show the improvements brought about by adding the Jacobi block to the Euler and
$R e$-dependent forms of the Van Leer-Lee-Roe matrix. Finally, Figure 12 shows the condition number for just the Re-dependent Van Leer matrix: it is $\mathrm{O}(1)$ over the entire $(M, R e)$ domain.

\section{Numerical Studies}

Below we give three examples of the action of NavierStokes preconditioners, including a case with turbulence modeling.

First, consider initial values consisting of a uniform field with a pressure perturbation in one central cell. The Mach number of the background flow is low $(0.1,0.01)$; the flow angle is $0^{\circ}$. Table 6 shows the number of iterations needed for 5 orders of magnitude of residual reduction; the scheme is first-order upwind Euler with centrally differenced viscous terms, and single-stage time-stepping. The grid consists of $10 \times 10$ square cells. It is seen that the convergence by the preconditioned schemes is hardly influenced by Mach or cell-Reynolds number, in contrast to the nonpreconditioned scheme.

The Van Leer/Jacobi and Chorin/Re preconditioner perform comparably, with some interesting differences. In the Euler limit the Van Leer matrix yields a lower condition number than the Chorin matrix (1 versus 2.6), explaining the somewhat faster convergence using the former. In the low-Re limit the situation is reversed, as the condition number for Van Leer/Jacobi is now a factor 5 larger than for Chorin/Re. For medium Reynolds number, $R e \approx 1$, the Chorin/Re matrix unexpectedly slows down convergence; this probably is the result of an inadequate choice of the switching function that connects the three branches of Eq. (11. It is clear that improvement is still possible here.

The second example is the computation of the development of a boundary layer on a flat plate $\left(M_{\infty}=\right.$ $\left.0.1, R e_{L}=4 \times 10^{4}\right)$. The cells right on the wall have an aspect ratio $\approx 1700$. The scheme is the same as in the first test. Figure 13 shows the steady flow field. As seen from the convergence histories in Figure 14, convergence without preconditioning is slow to begin with and keeps slowing down, while the preconditioned scheme has no convergence problem at all.

The third numerical test is the computation of the development of a turbulent boundary layer on the flat plate $\left(M_{\infty}=0.1, R e_{L}=3 \times 10^{5}\right)$. The SpalartAllmaras one-equation model is used for the turbulent transport. The convergence histories in Figure 15 show that the Van Leer/Jacobi preconditioner of Eq. (9) indeed achieves convergence, overcoming the double stiff- 


\begin{tabular}{||c|c|c|c||}
\hline Preconditioning & inviscid & $\begin{array}{c}\text { viscosity- } \\
\text { dominated }\end{array}$ & $\begin{array}{c}\text { acoustic } \\
\text { dominated }\end{array}$ \\
\hline None & $\frac{M+1}{\min (M,|1-M|)}$ & $\frac{\gamma}{R e}$ & $\frac{M+1}{M} R e$ \\
Chorin/Euler & $\mathcal{O}(1)$ & $\frac{1}{R e^{2}}$ & $\frac{1}{R e^{2}}$ \\
Van Leer/Euler & 1 & $\frac{1}{M^{2} R e^{2}}$ & $\frac{1}{R e^{2}}$ \\
Turkel/Euler & 1 & $\frac{1}{M^{2} R e^{2}}$ & $\frac{1}{R e^{2}}$ \\
Van Leer + Jacobi & 1 & $<\frac{1}{M^{2} R e^{2}}$ & $<\frac{1}{R e^{2}}$ \\
Van Leer/Re + Jacobi & 1 & $\mathcal{O}(1)$ & $\ll \frac{1}{R e^{2}}$ \\
Van Leer/Re & 1 & $\mathcal{O}(1)$ & $\mathcal{O}(1)$ \\
\hline
\end{tabular}

Table 5: Condition number produced by various 1-D Navier-Stokes preconditioners in different asymptotic regimes, based on PDE dispersion analysis. For a description of regimes and preconditioners see the main text. "Van Leer/Re" means: Van Leer-Lee-Roe preconditioner with Re-dependent $(1,1)$ element. The notation " $<$ ..." means: "lower than ...; analytic form hard to obtain."

ness due to the huge cell-aspect ratio $\left(\mathcal{R} \approx 10^{5}\right)$ and the large source term. Without preconditioning convergence is slow and eventually stalls.

\section{References}

[1] S. Abarbanel and D. Gottlieb, "Optimal time splitting for two and three dimensional Navier-Stokes equations with mixed derivatives." ICASE Report 80-6, 1980.

[2] S. R. Allmaras, "Analysis of semi-implicit preconditioners for multigrid solution of the 2-d compressible Navier-Stokes equations," AIAA Paper 95-1651-CP, 1995.

[3] A. Brandt, "Guide to multigrid development," in Multigrid Methods (W. Hackbush and U. Trottenberg, eds.), no. Lecture Notes in Mathematics 960, Springer Verlag, 1982.

[4] Y.-H. Choi and L. Merkle, "The application of preconditioning in viscous flows," Journal of Computational Physics, vol. 105, pp. 207-223, 1993.

[5] A. J. Chorin, "A numerical method for solving incompressible viscous flow problems," Journal of Computational Physics, vol. 2, 1967.

[6] D. L. Darmofal and P. J. Schmid, "The importance of eigenvectors for local preconditioning of the Euler equations," Journal of Computational Physics, vol. 127, no. 2, 1996.

[7] H. Deconinck and G. Degrez, "Monotone shock-capturing cell vertex schemes for the Euler and Navier-Stokes equations on unstructured grids," in Fifteenth International Conference on Numerial Methods in Fluid Mechanics, Springer, 1996.

[8] A. G. Godfrey, Topics on Spatially Accurate Methods and Preconditioning for the Navier-Stokes Equations with Finite-Rate Chemistry. PhD thesis, VPI \& SU, 1992.

[9] A. G. Godfrey, "Steps towards a robust preconditioning," AIAA Paper 94-0520, 1994.
[10] A. G. Godfrey, R. W. Walters, and B. van Leer, "Preconditioning for the Navier-Stokes equations with finite-rate chemistry," AIAA Paper 93-0535, 1993.

[11] D. Lee, Local Preconditioning of the Euler and NavierStokesEquations. PhD thesis, University of Michigan, 1994. In preparation.

[12] W.-T. Lee, Local Preconditioning of the Euler Equations. $\mathrm{PhD}$ thesis, University of Michigan, 1991.

[13] J. F. Lynn, Multigrid Solution of the Euler Equations with Local Preconditioning. PhD thesis, University of Michigan, 1995.

[14] J. F. Lynn and B. van Leer, "Multi-stage schemes for the Euler and Navier-Stokes equations with optimal smoothing," AIAA Paper 93-3355-CP, 1993.

[15] J. F. Lynn and B. van Leer, "A semi-coarsened multigrid solver for the Euler and Navier-Stokes equations with local preconditioning," AIAA Paper 95-1667-CP, 1995.

[16] J. F. Lynn, B. van Leer, and D. Lee, "Multigrid solution of the Euler equations with local preconditioning," in $F$ ifteenth International Conference on Numerial Methods in Fluid Mechanics, Springer, 1996.

[17] L. M. Mesaros, Multi-Dimensional Fluctuation Splitting Schemes for the Euler Equations on Unstructured Grids. $\mathrm{PhD}$ thesis, University of Michigan, 1995.

[18] L. M. Mesaros and P. L. Roe, "Multidimensional fluctuation-splitting schemes based on decomposition methods," AIAA Paper 95-1699, 1995.

[19] W. Mulder, "A new multigrid approach to convection problems," Journal of Computational Physics, vol. 83, pp. 303323, 1989.

[20] N. A. Pierce and M. B. Giles, "Preconditioning compressible flow calculatons on stretched meshes," AIAA Paper 96-0889, 1996.

[21] C. L. Reed, Low Speed Preconditioning Applied to the Compressible Navier-Stokes Equations. PhD thesis, University of Texas at Arlington, 1995. 


\begin{tabular}{||c|c|c|c|c|c|c||}
\hline \multirow{3}{*}{$R e_{h}$} & \multicolumn{6}{|c||}{$M$} \\
\cline { 2 - 7 } & \multicolumn{3}{|c|}{0.1} & \multicolumn{3}{c||}{0.01} \\
\hline & Unpc & VL/J & C/Re & Unpc & VL/J & C/Re \\
\hline $10^{6}$ & 679 & 86 & 91 & 5183 & 86 & 91 \\
$10^{4}$ & 679 & 86 & 92 & 5182 & 86 & 91 \\
$10^{2}$ & 675 & 87 & 117 & 5100 & 87 & 116 \\
1 & 1351 & 166 & 1830 & 5130 & 166 & 1858 \\
$10^{-2}$ & $50000(-4.74)$ & 194 & 133 & 5874 & 195 & 120 \\
$10^{-4}$ & $50000(-2.79)$ & 214 & 172 & $50000(-2.28)$ & 214 & 121 \\
$10^{-6}$ & $50000(-2.21)$ & 214 & 170 & $50000(-2.21)$ & 214 & 145 \\
\hline
\end{tabular}

Table 6: Number of iterations required for reduction of the density residual by a factor $10^{-5}$, (unless a smaller exponent is indicated in parentheses), in calculating the decay of a $10 M^{2} \%$ pressure perturbation in the center of a square domain $(10 \times 10$ cells, $\mathscr{A}=1)$. Discrete Navier-Stokes operator (first-order upwind/central differencing) with single-stage time marching. Unpc $=$ unpreconditioned; $\mathrm{VL} / \mathrm{J}=\mathrm{Van}$ Leer $+\mathrm{Jacobi} ; \mathrm{C} / R e=$ Chorin preconditioner modified to include Re-dependence.

[22] S. Ta'asan, "Canonical forms of multidimensional inviscid flows." ICASE Report 93-34, 1993.

[23] C.-H. Tai, Acceleration Techniques for Explicit Euler Codes. PhD thesis, University of Michigan, 1990.

[24] E. Turkel, "Preconditioned methods for solving the incompressible and low speed compressible equations," Journal of Computational Physics, vol. 72, 1987.

[25] E. Turkel, "Review of preconditioning methods for fluid dynamics." ICASE Report 93-42, 1993.

[26] E. Turkel, A. Fiterman, and B. van Leer, "Preconditioning and the limit to the incompressible flow equations." ICASE Report 93-42, 1993.

[27] E. Turkel, V. N. Vatsa, and R. Radespiel, "Preconditioning methods for low-speed flows," AIAA Paper 96-2460-CP, 1996.

[28] B. van Leer, W. T. Lee, and P. L. Roe, "Characteristic timestepping or local preconditioning of the Euler equations," in AIAA 10th Computational Fluid Dynamics Conference, 1991.

[29] B. van Leer, L. Mesaros, C.-H. Tai, and E. Turkel, "Local preconditioning in a stagnation point," in 12 th AIAA Computational Fluid Dynamics Conference, pp. 88-101, 1995. Also AIAA Report AIAA-95-1654-CP.

[30] S. Venkateswaran and C. L. Merkle, "Analysis of timederivative preconditioning for the navier-stokes equations," in 6th International Symposium on Computational Fluid Dynamics, 1995.

[31] G. Volpe, "On the use and accuracy of compressible flow codes at low Mach numbers," AIAA Paper 91-1662, 1991. 


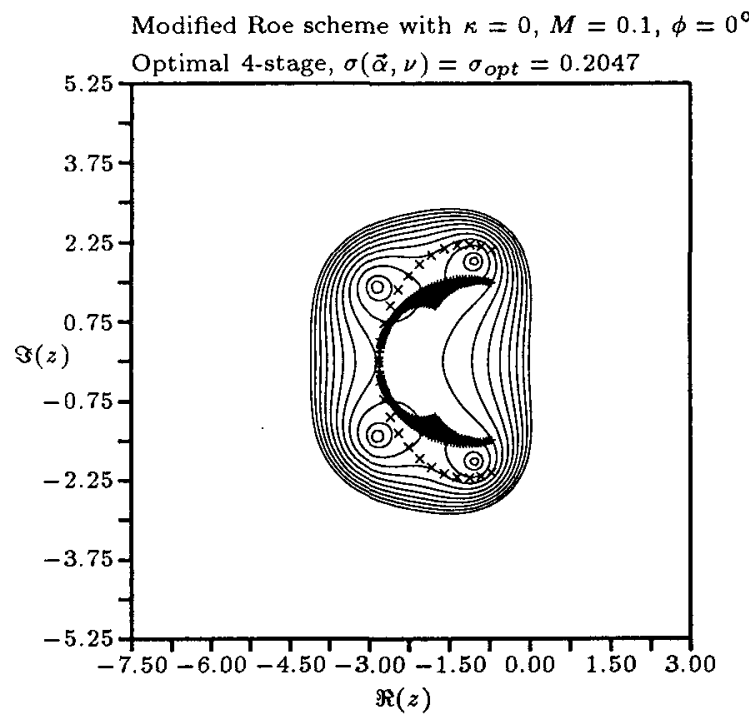

Figure 1: Design graph of optimal four-stage scheme for the $\kappa=0$ preconditioned Euler operator; $M=0.1, \phi=0^{\circ}$.

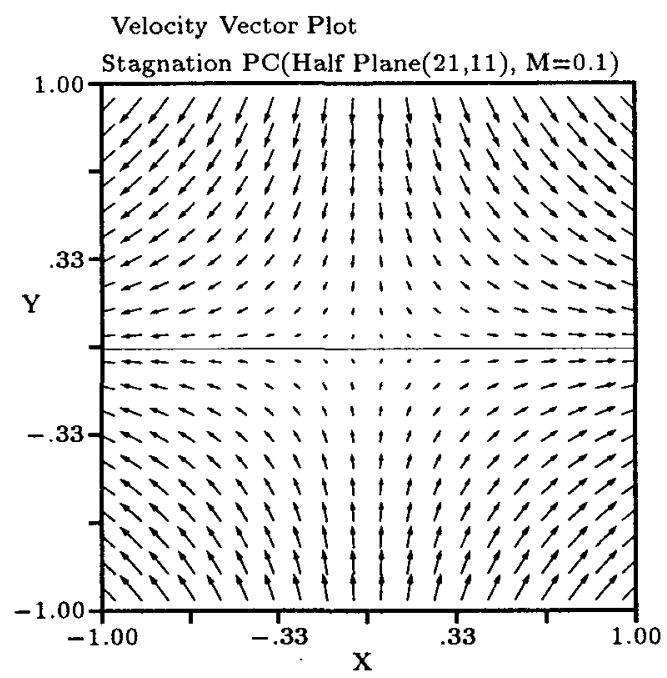

Figure 3: Flow field of half-plane stagnation flow, computed with the "stagnation preconditioner." The upper half is for $b_{0}=0$, the lower half for $b_{0}=1.5$

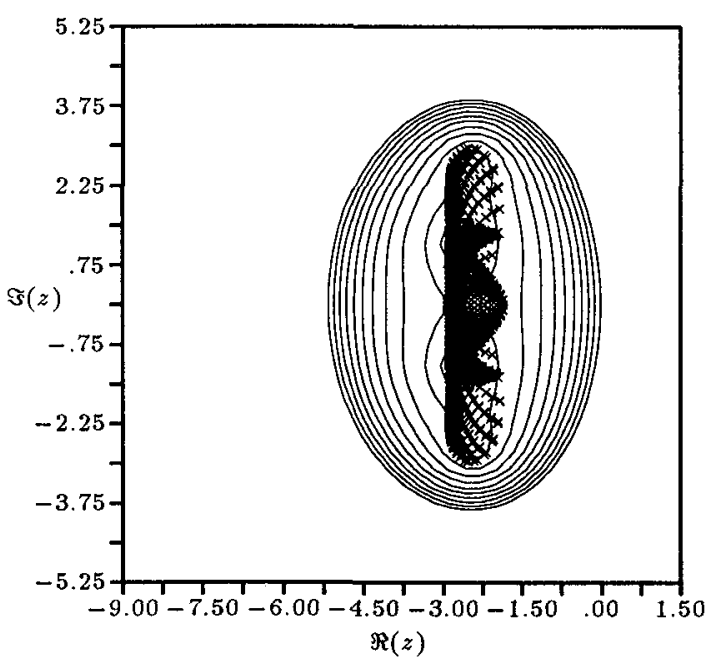

Figure 2: Design graph of optimal four-stage scheme for the first-order preconditioned Euler operator with explicit residual smoothing; $M=$ $0.1, \phi=45^{\circ}, \epsilon=0.1$.

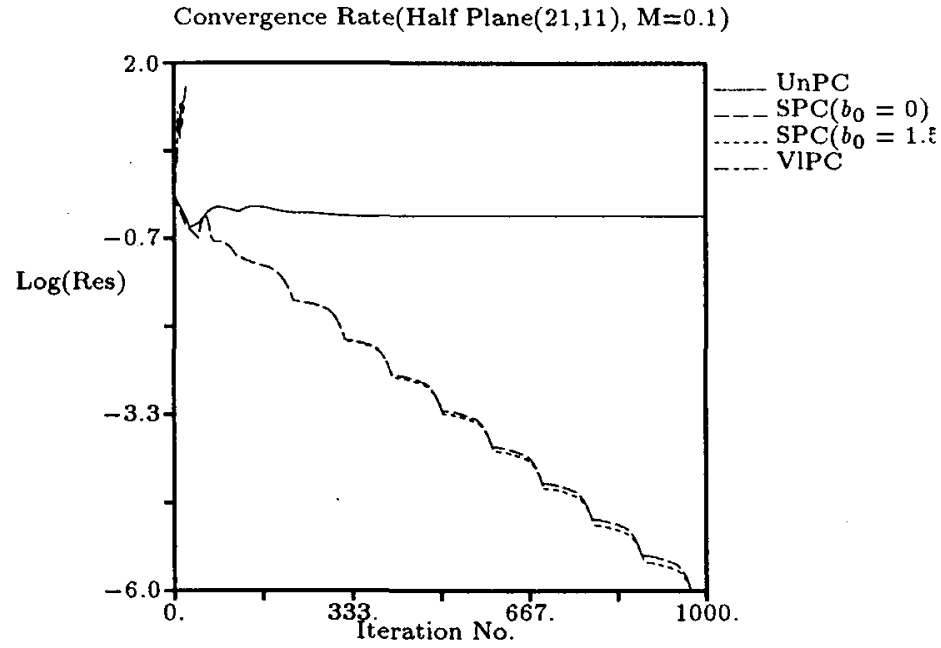

Figure 4: Residual history for half-plane stagnation-flow calculation; UnPC $=$ Unpreconditioned; SPC = Stagnation preconditioner; $\mathrm{VlPC}=$ Van Leer preconditioner. 


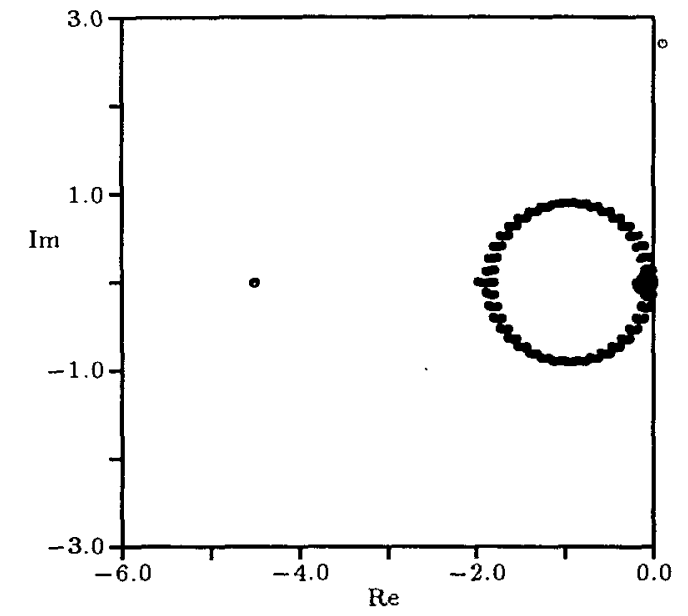

Figure 5: Fourier footprint for unpreconditioned Navier-Stokes scheme (first-order upwind/central) with turbulende modeling. $M=$ $0.1, R e_{h}=10^{6}, \frac{\left\|\mathbf{H}_{\mathrm{U}}\right\|}{\nu}=5 \times 10^{8}, \mathcal{A R}=10$.

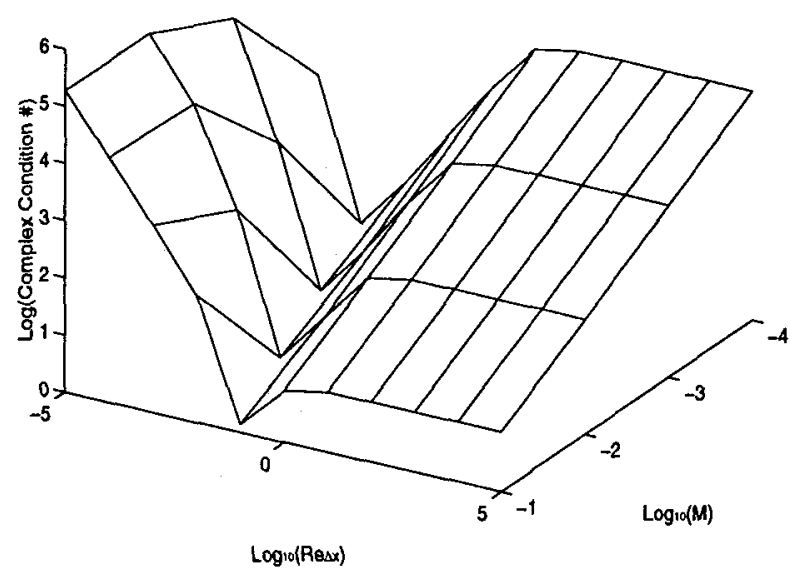

Figure 7: Condition number for 1-D NavierStokes equations without preconditioning.

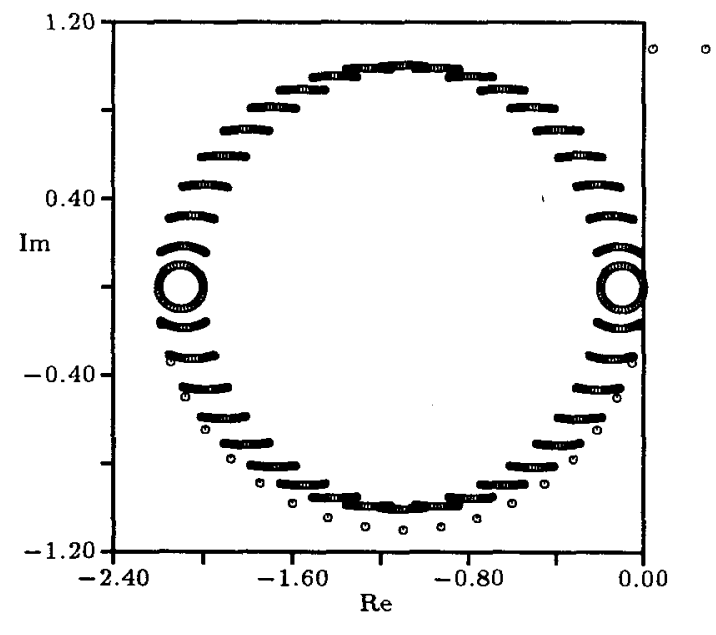

Figure 6: Same as in Figure 5, but after Van Leer/Jacobi preconditioning.

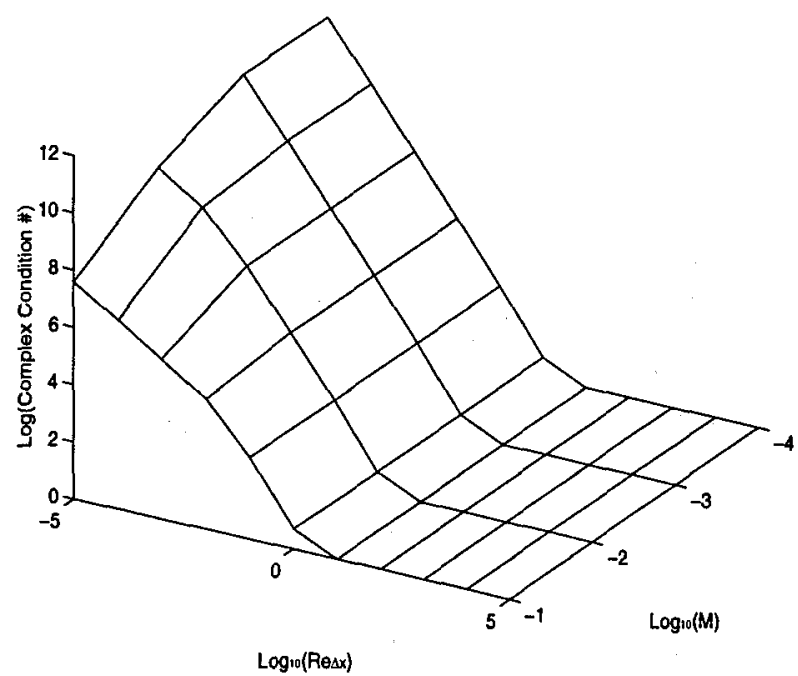

Figure 8: Same as Figure 7, using Van Leer's Euler preconditioner. 


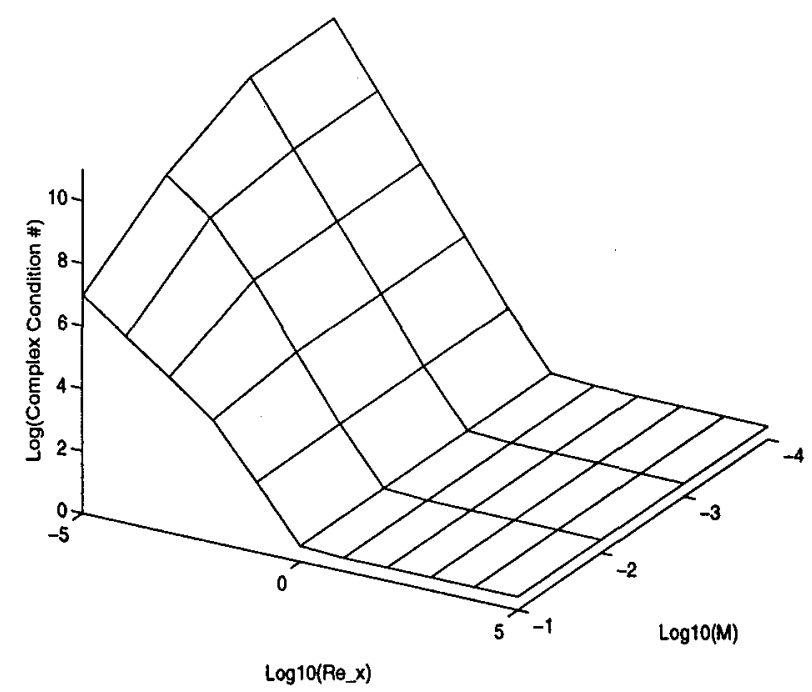

Figure 9: Same as Figure 7, using with Turkel's Euler preconditioner.

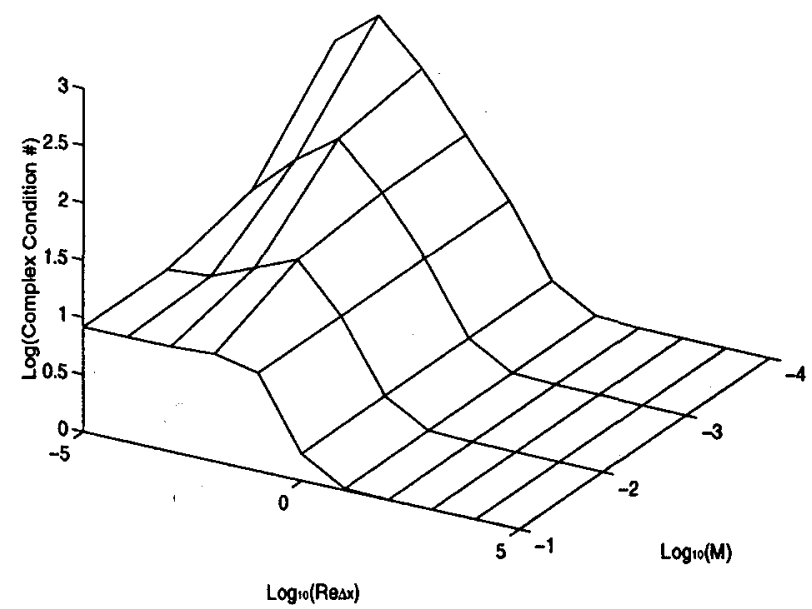

Figure 11: Same as Figure 7, using Van Leer's preconditioner modified to include $R e$ dependence, plus the viscous Jacobi block.

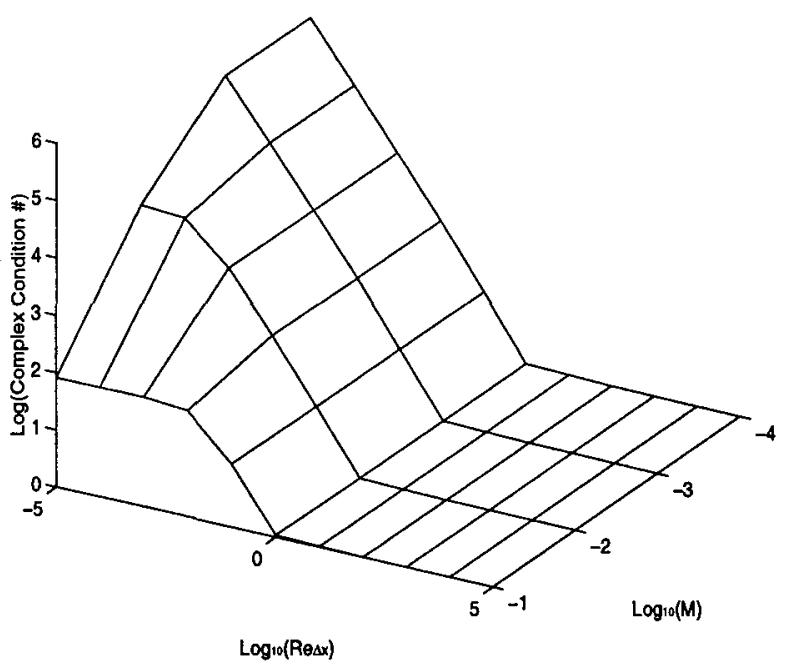

Figure 10: Same as Figure 7, using Van Leer/Jacobi preconditioning.

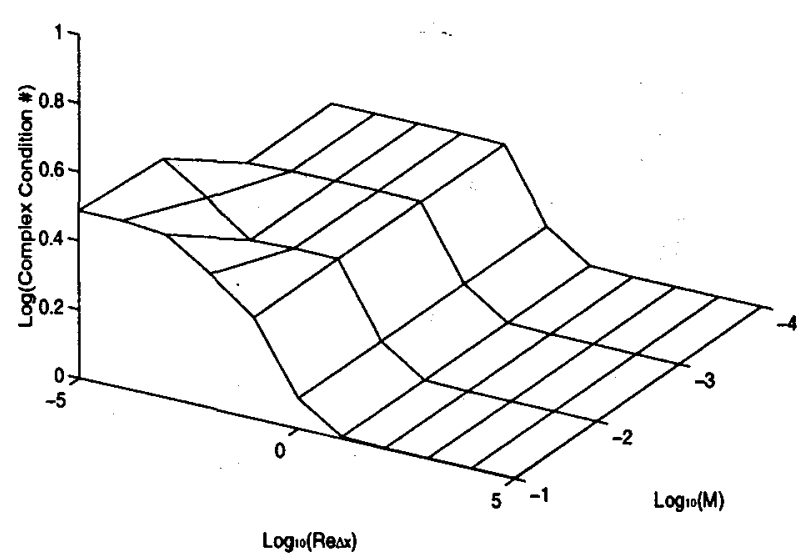

Figure 12: Same as Figure 7, using Van Leer's preconditioner modified to include $R e$ dependence. 


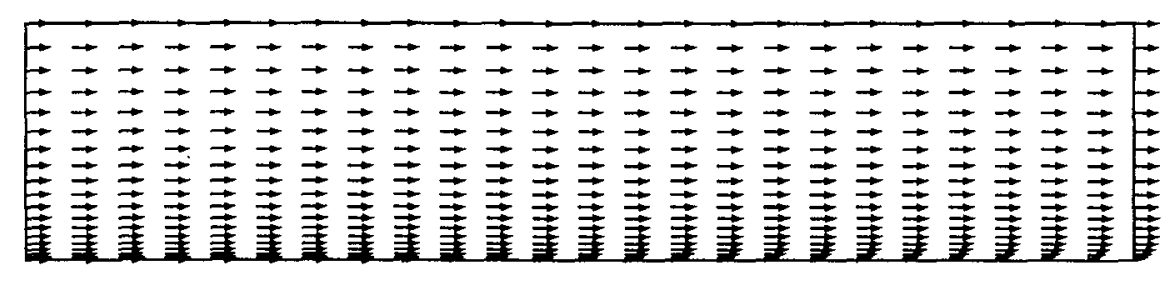

Figure 13: Velocity field for boundary-layer development on a flat plate. $M=0.1, R e_{L}=4 \times 10^{4}, 25 \times 20$ grid.

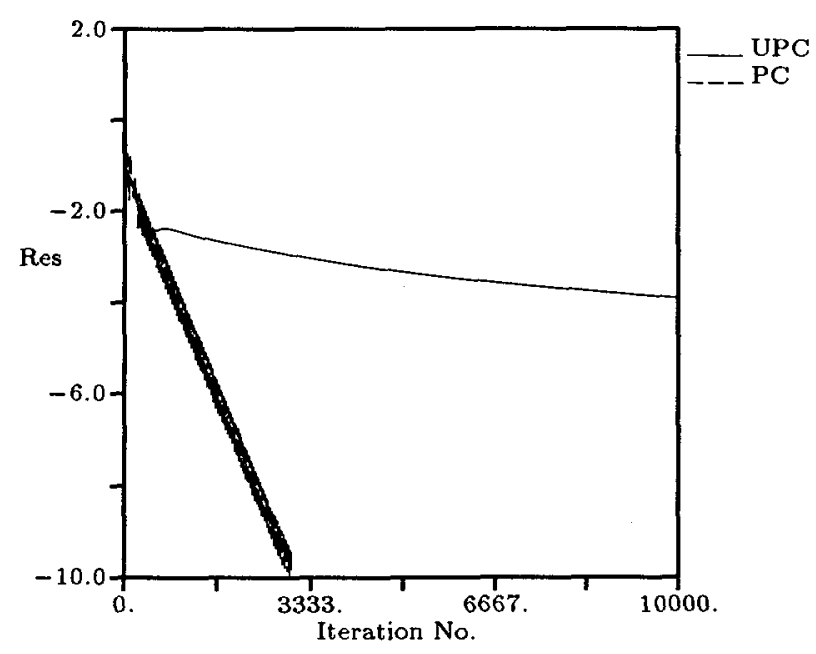

Figure 14: Convergence histories for the flatplate boundary-layer calculations. $\quad M=0.1$, $R e_{L}=4 \times 10^{4}, 25 \times 20$ grid. UPC $=$ unpreconditioned, $\mathrm{PC}=$ Van Leer/Jacobi preconditioner.

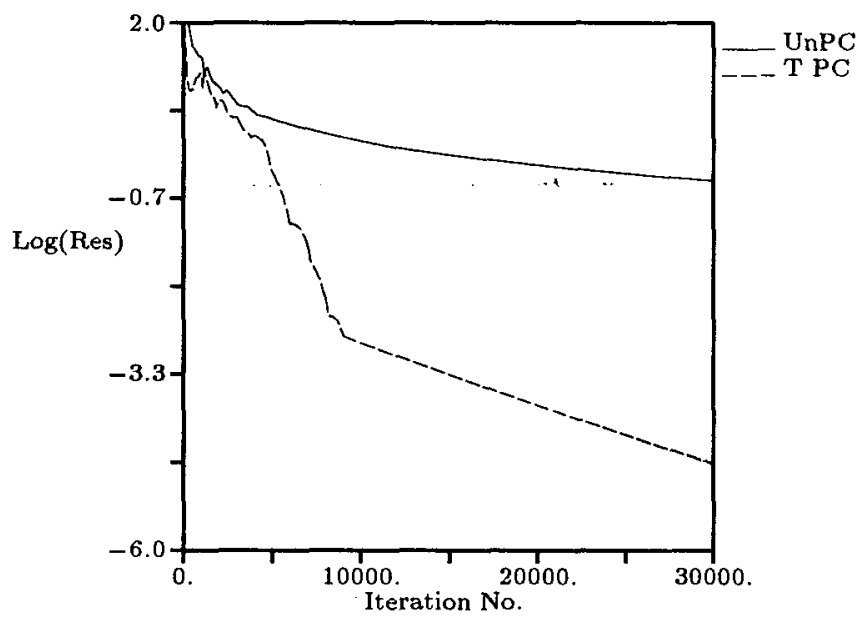

Figure 15: Convergence histories for the flatplate boundary-layer calculations with turbulence modeling. $M=0.1, R e_{L}=3 \times 10^{5}, 25 \times 50$ grid. $\mathrm{UnPC}=$ unpreconditioned; $\mathrm{T} \mathrm{PC}=$ Van Leer/Jacobi preconditioner including turbulent source-term Jacobian. 